\title{
In-center hemodialysis and COVID 19 infection
}

\author{
Seyed Seifollah Beladi Mousavi ${ }^{\circledR}$, Shahla Ahmadi Halili ${ }^{*}{ }^{\circledR}$, Asieh Aref ${ }^{\circledR}$ \\ Chronic Renal Failure Research Center, Ahvaz Jundishapur University of Medical Sciences, Ahvaz Iran
}

Correspondence to:

Shahla Ahmadi Hallili, Email:

Ahmadihallili@gmail.com

Received: 4 July. 2020

Accepted: 13 Dec. 2020

ePublished: 29 Dec. 2020

Keywords: COVI D-19, SARSCoV-2, Hemodialysis, End-stage kidney disease

Citation: Beladi Mousav SS, Ahmadi Halili S, Aref A. In-center hemodialysis and COVID 19 infection. J Prev Epidemiol 2020;5(2):e32. doi: 10.34172/jpe.2020.32

\section{Key point}

It seems that end-stage kidney disease (ESKD) patients undergoing maintenance hemodialysis (HD) are at higher risk for COVID 19 infection because they have frequent contact with the health care systems. There is not any specific HD center outside of hospital for patients with COVID 19 infection in many developing countries and therefore it is difficult to isolate HD patients who have COVID 19 infection in an isolated room for dialysis. Therefore we are suggested that in developing countries like our country, HD patients who have suspected or confirmed COVID 19 infection and who are not severely ill can be dialyzed in their HD centers. However use of personal and patient protective equipment in the HD unit is mandatory.

$\mathrm{T}$ he outbreak of highly contagious COVID-19, a new strand of the coronavirus family, has become a global epidemic of diseases, which poses a serious threat to human health, especially for elderly and those with underlined diseases (1-3).

It is not known whether end-stage kidney disease (ESKD) patients are at higher risk for COVID-19 infection compared to the general population; however, it seems theoretically that these patients particularly those who undergoing maintenance intermittent hemodialysis (HD) are at higher risk for this infection because they have frequent contact with the health care systems during HD session $(2,3)$.

In addition, ESKD patients who undergoing intermittent HD are particularly vulnerable to severe COVID-19 and poor outcomes due to high rates of complications and co-morbidities, such as diabetes and hypertension, among this population (4-7). What should be we do for HD patients who have confirmed or suspected COVID 19?

According to the Centers for Disease Control, American Society of Nephrology and International Society of Nephrology guidelines, ESRD patients with SARSCoV-2 should be co-localized on a floor or intensive care unit to minimize contact of patients who have COVID-19 with others. In addition, according to these guidelines, patients with suspected COVID-19 who have mild respiratory symptoms and who are not critically ill should also be dialyzed in an isolation room rather than an inpatient dialysis center $(8,9)$. Unfortunately, these recommendations are not possible to perform in many developing countries including our country.

The most common renal replacement therapy in Iran is HD which is mainly presence of nursing staff as conventional intermittent HD two or three times per week. Approximately $3.5 \%$ of our end-stage renal disease (ESRD), patients undergoing continuous ambulatory peritoneal dialysis and home hemodialysis, is not performed in our country $(4,10,11)$.

In-center HD limits our ability to perform physical isolation for infection control among HD patients in the pandemic of COVID 19 and therefore it is difficult or impossible to perform above recommendations for HD patients.

Firstly, the clinical manifestations of patients who have SARS-CoV-2 infection including fever and/or respiratory tract symptoms (eg, cough, dyspnea) are not specific and these syndromes can occur with other viral respiratory illnesses; therefore diagnosis of COVID 19 infection according to the sign and symptoms of the patients is difficult and cannot be definitively made without microbiologic testing (3). On the other hand, most of the patients who have COVID 19 including ESRD patients are asymptomatic. For example according to the report of Chinese Center for Disease Control and Prevention which evaluated performed in hospitals or HD centers in the 
approximately 44500 confirmed SARS-CoV-2 infections, $81 \%$ of the patents did not have any symptoms or only had mild respiratory symptoms $(3,8)$.

The results of $\mathrm{Ma}$ et al study performed among HD patients showed that the symptoms of most of the HD patients with COVID 19 are mild (12).

Secondly, laboratory tests for COVID 19 infection including direct detection of SARS-CoV-2 RNA by nucleic acid amplification tests, primarily reverse transcription polymerase chain reaction (RT-PCR) or serologic tests which detect IgG and IgM antibodies to SARS-CoV-2 in the blood, are very expensive and are not also readily available. In addition false positive and negative of these tests are high and based on these tests, we cannot confirm patients who have or don't have COVID 19 infection definitely (2).

Thirdly, it is also important to note that there is not any specific HD center outside of hospital for patients with COVID 19 infection in many developing countries including our country and therefore it is difficult to isolate HD patients who have COVID 19 infection in an isolation room for dialysis.

According to the above important notes, we suggested that in developing countries like our country, HD patients who have suspected or confirmed COVID 19 infection and who are not severely ill can be dialyzed in their HD centers. However use of personal and patient protective equipment in the HD unit is mandatory. It is also suggested that HD patients with signs and symptoms of respiratory infection (eg, fever, cough) should be recognized before they enter the treatment area and if possible. Likewise, these patients should be dialyzed in a separate room with the closed door (4-9).

If a separate room is not available in HD center, patients with respiratory symptoms who have suspected or confirmed COVID 19 infection should be dialyzed in corner or end-of-row stations and should be separated from other patients in all directions by at least two meters (5-7).

In $\mathrm{HD}$ centers that have hepatitis $\mathrm{B}$ isolation rooms, the isolation room can also be used for patients who have suspected or confirmed COVID 19 infection only if the room is not needed to dialyze hepatitis $B$ infected patients (4-8).

Authors' contribution

SSBM and SAH prepared the primary draft. AA edited the paper. All authors read and signed the final paper.
Conflicts of interest

The authors declare that they have no competing interests.

Ethical considerations

Ethical issues (including plagiarism, data fabrication, double publication) have been completely observed by the authors.

Funding/Support

Chronic Renal Failure Research Center of Ahvaz Jundishapur University of Medical Sciences supported the study.

References

1. World Health Organization. Director-General's remarks at the media briefing on 2019-nCoV on 11 February 2020. Available from: https://www.who.int/dg/speeches/detail/who-directorgeneral-s-remarks-at-the-media-briefing-on-2019-ncov-on11-february-2020. Accessed February 12, 2020.

2. World Health Organization. Novel Coronavirus (2019-nCoV) technical guidance. Available from: https://www.who.int/ emergencies/diseases/novel-coronavirus-2019/technicalguidance. Accessed February 14, 2020.

3. Wu Z, McGoogan JM. Characteristics of and Important Lessons From the Coronavirus Disease 2019 (COVID-19) Outbreak in China: Summary of a Report of 72314 Cases From the Chinese Center for Disease Control and Prevention. JAMA 2020. doi: 10.1001/jama.2020.2648.

4. Kliger AS, Silberzweig J. Mitigating Risk of COVID-19 in Dialysis Facilities. Clin J Am Soc Nephrol 2020; 15:707. doi: 10.2215/CJN.03340320

5. Centers for Disease Control and Prevention. Interim Additional Guidance for Infection Prevention and Control Recommendations for Patients with Suspected or Confirmed COVID-19 in Outpatient Hemodialysis Facilities. Available from: $\quad$ https://www.cdc.gov/coronavirus/2019-ncov/hcp/ dialysis.html. Accessed April 8, 2020.

6. Ma Y, Diao B, Lv X, Zhu J, Liang W, Liu L, et al. 2019 novel coronavirus disease in hemodialysis (HD) patients: Report from one HD center in Wuhan, China. Available from: https:// www.medrxiv.org/content/10.1101/2020.02.24.20027201v2.

7. Kliger AS, Silberzweig J. Mitigating Risk of COVID-19 in Dialysis Facilities. Clin J Am Soc Nephrol. 2020; 15:707.

8. MaY, Diao B, LvX, Zhu J, Liang W, Liu L, BuW et al. 2019 novel coronavirus disease in hemodialysis (HD) patients: Report from one HD center in Wuhan, China. Available at: https:// www.medrxiv.org/content/10.1101/2020.02.24.20027201v2

9. Centers for Disease Control and Prevention. Interim Additional Guidance for Infection Prevention and Control Recommendations for Patients with Suspected or Confirmed COVID-19 in Outpatient Hemodialysis Facilities. https://www. cdc.gov/coronavirus/2019-ncov/hcp/dialysis.html. Accessed April 8, 2020. 